\title{
Les systèmes alimentaires aux défis de la crise de la Covid-19 en Afrique : enseignements et incertitudes
}

\author{
Sandrine Dury ${ }^{1,2,3, *}$, Arlène Alpha ${ }^{2,3}$, Nadine Zakhia-Rozis ${ }^{4}$ et Thierry Giordano ${ }^{5}$ \\ ${ }^{1}$ Cirad, Département Environnements et Sociétés, Montpellier, France \\ 2 Moisa, Univ Montpellier, Cirad, INRAE, Ciheam-Iamm, L'institut Agro IRD, Montpellier, France \\ 3 Cirad, UMR Moisa, 34398 Montpellier, France \\ ${ }^{4}$ Cirad, Département Performances des systèmes de production et de transformation tropicaux, Montpellier, France \\ ${ }^{5}$ Cirad, Direction de l'impact et du marketing de la science, Montpellier, France
}

\begin{abstract}
Résumé - La crise liée au SARS-CoV2 (syndrome respiratoire aigu sévère-coronavirus 2) a donné lieu à une profusion de documents et webinaires sur la sécurité alimentaire au niveau international, ce qui tend à brouiller la compréhension des dynamiques à l'œuvre sur le terrain. Cet article se propose de faire le point sur la situation des secteurs agricole et agroalimentaire, à partir des informations relayées par un réseau d'experts du Cirad et de leurs partenaires dans une diversité de pays en Afrique subsaharienne. Mises en perspective avec les rapports des organisations internationales, ces informations permettent de dresser un constat nuancé. Si le commerce mondial des produits de base et la production agricole dans les pays du Sud ont été relativement peu affectés par les mesures de restriction prises pour limiter la propagation du virus, certaines filières de produits périssables ont rencontré des difficultés pour écouler leurs produits. Mais surtout, la crise a mis en exergue la déconnexion entre le monde de la production agricole, du commerce alimentaire et la situation des consommateurs précaires, qui dans les villes et dans le secteur informel ont pris de plein fouet l'arrêt des activités économiques. Cette crise sanitaire invite à repenser les mesures de gestion de la crise, tant à court terme qu'à plus long terme, avec une approche intégrée des systèmes alimentaires pour renforcer leur résilience et soutenir les stratégies d'adaptation des acteurs.
\end{abstract}

Mots clés : Covid-19 / agriculture / systèmes alimentaires / sécurité alimentaire / pays en développement

\begin{abstract}
The COVID-19 crisis is challenging the food systems in Africa. Many documents have been published and webinars held on the impact of the COVID-19 crisis on food security at international levels, which tend to blur our understanding of actual dynamics on the ground. Based on a network of researchers from Cirad and its partners in a wide diversity of countries in Sub-Saharan Africa, this paper attempts to take stock of what is happening in the food and agriculture sector. The information provided allow for a more nuanced analysis when compared to international organizations' reports. While lockdown and social distancing measures have only mildly affected world trade and production of staple food, some perishable products have been wasted, unable to reach markets. The crisis emphasizes the disconnection between food production and trade and the dire situation of many poor consumers, in cities, holding informal jobs, who have been hit very hard. The crisis should help imagining short- and long-term measures to support food systems resilience along an integrated approach and adaptation strategies already developed.
\end{abstract}

Keywords: COVID-19 / agriculture / food systems / food security / developing countries

\section{Introduction}

La crise sanitaire mondiale a secoué, et secoue encore, l'ensemble de la planète et les conséquences humaines, économiques et sociales ne sont encore claires ni dans leur

\footnotetext{
* Auteur de correspondance : sandrine.dury@cirad.fr
}

nature, ni dans leur intensité, ni dans leur durée. Les pronostics initiaux sur la propagation de la maladie ont tous été remis en cause les uns après les autres, et les décideurs politiques ont dû s'adapter et prendre des mesures en urgence. Les conséquences sur la sécurité alimentaire et les systèmes alimentaires de l'épidémie de Covid-19 et des mesures prises pour l'enrayer sont tout aussi incertaines à court, moyen ou long terme. Rapidement, depuis le mois de mars 2020 et jusqu'à 
maintenant, de nombreuses données, analyses et communications ont été produites, notamment par les services des Nations Unies en charge de l'alimentation et de l'agriculture (FAO, PAM, FIDA), par l'Institut international de recherche sur les politiques alimentaires (IFPRI), ou encore par l'Organisation internationale du travail (2020) sur les conséquences de la crise sur la sécurité alimentaire et nutritionnelle (l'accès de tous à l'alimentation suffisante et saine; CSA, 2012) ou sur les systèmes alimentaires (qui incluent les acteurs de la production, transformation, commercialisation de l'alimentation, et les relations entre eux; HLPE, 2017). Les travaux de ces organismes sont souvent utilisés dans une perspective globale, informant sur les conséquences de la crise au niveau mondial ou dans les grandes régions du monde.

La présente contribution entend apporter un complément à ces analyses fort utiles en donnant à voir des réalités de terrain dans une diversité de contextes, essentiellement en Afrique. Nous présentons le résultat d'un travail de mise en réseau d'experts du Cirad et de leurs partenaires travaillant sur les systèmes alimentaires dans différents pays d'Afrique. En tant que chercheurs engagés, témoins d'un évènement sans précédent, plusieurs d'entre eux ont publié des textes sur différents supports (journaux, série spéciale du Cirad sur Covid et sécurité alimentaire), organisé ou participé à des séminaires sur le sujet avec différentes parties prenantes: chercheurs, ONG, politiques (ministères), bailleurs publics ou privés du développement (Agence française de développement, Bill and Melinda Gates Foundation, Banque mondiale). La collecte d'information pour ces analyses a porté sur la période de mars à fin juin 2020. Elle s'est appuyée sur plusieurs méthodes : entretiens qualitatifs avec des opérateurs de systèmes alimentaires avec lesquels le Cirad entretient une relation de confiance de longue date, enquêtes légères sur divers points de vente, relevés de prix, veille documentaire. Vu l'absence de recul historique et l'empirisme de l'approche, il convient de prendre cette contribution comme une série d'hypothèses qui n'engagent que les auteurs de ce texte, mais dont la valeur d'expérience nous paraît importante. Les analyses portent sur une période de quatre mois, de mars à juin 2020.

L'idée centrale que nous défendons est que la production agricole, qui prend du temps entre les semis et les récoltes, a globalement été relativement peu affectée durant la période d'étude, alors que la partie aval de la production a été fortement affectée. Dépendant fortement d'une main-d'œuvre familiale, dans un contexte encore très rural, l'agriculture africaine semble avoir peu souffert d'un manque de main-d'œuvre lié aux restrictions de déplacement. Nous n'avons pas eu connaissance de témoignage dans ce sens, sauf peut-être en Afrique du Sud où le modèle agro-industriel est plus développé. Les circuits de distribution ont été partiellement interrompus mais souvent rétablis au bout de quelques semaines pour assurer l'écoulement des vivres de première nécessité. Globalement, la partie amont du système alimentaire a tenu et a été appuyée par les gouvernements. En revanche, la partie aval, avec les consommateurs et les petits transformateurs et commerçants, a été touchée de plein fouet par les mesures de confinement, la récession économique et les pertes de salaires en conséquence. En retour, dans certaines filières agricoles comme celles de produits périssables (lait et fruits et légumes), les producteurs ont eu, ont et auront encore des difficultés à écouler leurs produits du fait d'une contraction de la demande, et ont enregistré des pertes importantes de récoltes et de revenus, dans des contextes de stockage et de transformation limités. Ils vont certainement devoir ajuster leur production pour les prochaines saisons. Nous présentons ci-après le détail de cette argumentation.

Il faut noter la difficulté de tenir un discours uniforme tant les situations particulières sont nombreuses et tant la situation est inédite. Il existe également une multiplicité de prises de position publique de différents acteurs, "à chaud », et souvent sur un mode catastrophique, qui dans certains cas révèle effectivement une situation alarmante (ou menaçant de l'être), mais qui dans d'autres fait partie de la rhétorique classique du plaidoyer ou du lobbying des «parties prenantes». Quelques positionnements ont été plus mesurés, et nous avons voulu leur donner une place, car, dans ce brouhaha médiatique, ils passent souvent inaperçus. Ces positionnements reflètent peut-être un certain relativisme dans des situations africaines, en particulier lorsque les personnes font face à de multiples risques au quotidien et ont une «habitude» à y faire face. Ces capacités d'adaptation (que l'on peut questionner par ailleurs tant les conditions de vie moyennes sont non satisfaisantes) amènent certainement certains observateurs à relativiser les effets possibles et/ou réels de la pandémie actuelle. Nous avons essayé de naviguer entre ces deux écueils de catastrophisme et de relativisme. Les différentes publications sur l'impact de la Covid-19 dans le monde et les informations collectées à travers les différents réseaux de chercheurs du Cirad semblent converger vers les traits saillants suivants.

\section{Une situation mondiale marquée par une crise de la demande}

\subsection{Un bon niveau de production et des stocks agricoles}

Après plusieurs alertes sur la production des denrées de base, il est rapidement apparu que la production des denrées de base échangeables (riz, maïs, soja, blé) était d'un niveau élevé cette année. Après des craintes initiales sur le commerce mondial, les échanges internationaux de produits alimentaires n'ont été que peu perturbés par les politiques nationales. Contrairement aux déplacements de personnes, les transports de ces denrées n'ont pas été interrompus, des dispositions ayant été prises pour assurer la continuité des approvisionnements. Le système alimentaire mondial reliant les principaux pays producteurs et exportateurs (Vietnam, Chine, Russie, Brésil, France...) aux pays consommateurs (pays européens, pays africains, selon les produits) a donc continué de fonctionner. Les restrictions aux exportations qui ont eu lieu dans certains pays (par exemple au Vietnam ou en Ukraine) n'ont pas duré et les chaînes internationales n'ont pas été rompues (Laborde Debucquet et al., 2020). Les ports africains sont toujours approvisionnés en blé et en riz, les ports européens et chinois en soja brésilien pour l'élevage. La situation pour les produits périssables, échangés sur de plus courtes distances, de même que dans certains pays, est différente comme on le verra par la suite.

Les stocks mondiaux des denrées de base sont également importants, ce qui a permis de limiter les fluctuations et la hausse des prix. D'après la FAO, la production de céréales dans le monde devrait atteindre un niveau record et porter le rapport 
stocks/utilisation à son plus haut niveau en 20 ans (http://www. fao.org/worldfoodsituation/csdb/fr/, consulté le 7/7/2020).

Cette situation est donc très différente de celle de 2008, qui était une crise de l'offre alimentaire, où les prix internationaux des denrées alimentaires de base et du pétrole s'étaient envolés (Daviron, 2012; Mendez Del Villar, 2020). Néanmoins, la crise alimentaire est bien réelle pour de nombreuses populations, comme l'ont souligné très tôt les organisations internationales. Plusieurs observateurs remarquent que pour les produits de base au moins, et au niveau mondial, il n'y a pas de «crise de l'offre », ce n'est «pas une crise agricole». En revanche, ces mêmes observateurs s'accordent pour parler de « crise de la demande».

\subsection{Une augmentation de la précarité dans le monde entier}

Les mesures généralisées de restriction des déplacements, plus ou moins intenses selon les pays, ont mis à l'arrêt de nombreuses activités économiques, et fait perdre leur emploi à des millions de personnes, dont beaucoup, aux revenus modestes, sombrent dans l'insécurité alimentaire. Cela concerne le monde entier, pays riches comme pays pauvres, et cela est décrit dès avril par différents observateurs : par exemple en Inde (Dorin, 2020), aux États-Unis (DeParle, 2020), en France, où la distribution alimentaire est réalisée par des associations qui témoignent de la hausse de la précarité (Bricas et al., 2020). De ce point de vue, la crise est à la fois un révélateur et un facteur aggravant des inégalités socioéconomiques préexistantes (FMI, 2020 ; IPES Food, 2020). Laborde et al. (2020) ont publié en avril des simulations sur l'augmentation du nombre de pauvres dans le monde en conséquence de la récession économique dans les pays développés et émergents, phénomène qui se propage progressivement aux pays pauvres. Ils estiment que 140 millions de personnes supplémentaires pourraient tomber dans l'extrême pauvreté, dont 80 millions en Afrique sub-saharienne, région la plus touchée. Le rapport sur la nutrition mondiale 2020 insiste sur les inégalités persistantes en termes de nutrition dans le monde (Independent Expert Group, 2020) : une personne sur neuf dans le monde a faim ou est sousalimentée, une sur trois est en surpoids ou obèse. Les personnes en état de malnutrition par carence ou par excès (obésité) sont encore plus sensibles à la Covid.

\subsection{La superposition de crises met en danger la sécurité alimentaire}

Dans les pays les plus sensibles et les plus dépendants de l'aide alimentaire internationale, la crise Covid se superposant à d'autres problèmes (conflits armés, criquets...), le nombre de personnes en insécurité alimentaire grave pourrait augmenter de plusieurs dizaines de millions, et la situation Covid perturbe aussi l'acheminement de l'aide alimentaire. Dès le mois d'avril 2020, avant la crise, le Réseau de prévention des crises alimentaire (RPCA) alertait sur le fait qu'en Afrique de l'Ouest et au Sahel, 17 millions de personnes risquaient d'avoir besoin d'aide alimentaire à l'été 2020. Ce chiffre pourrait augmenter de 51 millions de personnes en raison de la Covid-19 (RPCA, 2020).
Le système alimentaire mondial semble avoir globalement « tenu » à court terme du côté de la production et des échanges internationaux, mais les problèmes d'emplois et de revenus résultant des mesures de confinement provoquent une forte aggravation de l'insécurité alimentaire et nutritionnelle dans tous les pays. La récession économique générale et l'augmentation du chômage et de la pauvreté liée à la situation Covid expliquent largement la flambée de l'insécurité alimentaire nutritionnelle en cours et possiblement à venir. Par exemple, le Fonds monétaire international (FMI, 2020) prévoyait, en juin 2020, que la récession mondiale s'élèverait à $-4,9 \%$ pour l'année 2020. Cela équivaut à environ 300 millions d'emplois à plein temps perdus. Selon la Banque africaine de développement (2020), le PIB se contracterait en 2020 de 1,7 à $3,4 \%$ en Afrique, ce qui correspondrait à une augmentation de l'ordre de 25 à 30 millions de personnes sans emploi, et 28 à 49 millions de personnes poussées dans l'extrême pauvreté. À partir de ces chutes possibles du PIB, le rapport 2020 sur «l'état de la sécurité alimentaire et nutritionnelle dans le monde», estime que, suite à la crise, le nombre de personnes en situation d'insécurité alimentaire pourrait augmenter de 83 à 132 millions suivant les scénarios (FAO et al., 2020).

Bien que les situations particulières des pays soient très contrastées, selon les mesures prises, les produits concernés, les acteurs des filières, etc., quelques constats récurrents peuvent être faits.

\section{Un système de production qui se heurte aux contraintes de consommation dans les pays du Sud}

\subsection{Des problèmes de débouchés et de revenus pour les producteurs}

Les mesures de confinement et de restriction de circulation ont touché en priorité les capitales et les villes secondaires. L'approvisionnement des marchés urbains en a été fortement affecté. Certaines villes comme Abidjan ou Conakry ont été placées en quarantaine; certains marchés urbains de plein air ont été fermés, comme à Ouagadougou. Ainsi, les producteurs de produits périssables ont été durement frappés, se retrouvant sans débouchés pour écouler leur production: plusieurs témoignages concordent par exemple sur le secteur laitier à Madagascar (Audouin et al., 2020) ou en Afrique de l'Ouest (Vall et al., 2020a, 2020b) ou concernant la mise en marché des légumes au Laos (Lao Farmer Network, 2020). «Dans certains pays producteurs de pomme de terre (Guinée, Nigéria, Sénégal...) le pic de production a coïncidé avec les restrictions de circulation et une partie significative des récoltes (entre 20 et $50 \%$ selon les pays) a été perdue en raison du manque d'infrastructures de stockage et de moyens logistiques » (MAA, 2020). Au-delà des pertes aux champs ou dans les entrepôts, les producteurs de pommes de terre de Guinée en viennent à brader leurs produits, faute de pouvoir les exporter vers les pays voisins ou les écouler vers les marchés habituels, mettant en péril leurs capacités de remboursement des prêts contractés et la prochaine campagne (AFDI, 2020; FPFD, 2020).

La fermeture des frontières a inquiété également les pasteurs d'Afrique de l'Ouest (APESS, 2020). L'impossibilité 
de faire transhumer les troupeaux vers des zones de pâturage entraîne des dommages irréversibles pour les animaux, pour les pasteurs et leurs familles, ainsi que pour les agro-éleveurs sédentaires qui ont confié leurs animaux à ces pasteurs. De plus, un trop long séjour dans les régions de savane provoque à la fois des problèmes de dégradation des sols, et surtout des tensions avec les agriculteurs. Ceci étant, malgré les fortes craintes initiales, et peut-être grâce à ce plaidoyer réalisé par les organisations d'éleveurs et pasteurs, il semble que, rapidement, les frontières aient été ré-ouvertes pour éviter les drames évoqués précédemment.

Il existe une inquiétude sur les exportations africaines de produits agricoles sur le marché international. La baisse de la consommation de chocolat, par exemple durant les fêtes de Pâques en Europe et aux États-Unis, l'augmentation des stocks en Europe, une obligation de garder des stocks dans les pays de production et une éventuelle baisse des cours, inquiètent les organisations de producteurs de cacao (Ruf, 2020). Les producteurs de coton s'inquiètent également de la baisse de la demande (mise à l'arrêt des usines textiles), et les prix aux producteurs, par exemple au Mali, ont été revus à la baisse (Dugué, 2020).

\subsection{Des difficultés de production relativement limitées}

Plusieurs alertes ont été lancées concernant l'accès aux intrants, semences et engrais, de même que sur la disponibilité de la main-d'œuvre. De fait, ces préoccupations semblent plutôt celles des agriculteurs spécialisés, et concernent peu les agricultures familiales qui sont très faiblement dépendantes des intrants commerciaux. Par ailleurs, le cours du pétrole historiquement bas devrait se traduire par une baisse du coût des engrais et des frais de transport. Ainsi, les spécialistes ne montrent pas une inquiétude forte sur les conditions de production (Dugué, 2020). En revanche, les effets sur les prochaines saisons de production pourraient être particulièrement importants, suite notamment à la baisse de revenu des producteurs, ce qui pourrait limiter leur capacité d'accès aux intrants, et à la résurgence de maladies dont le contrôle a été perturbé par la pandémie (FAO, 2020).

Par ailleurs, la plupart des témoignages insistent sur le fait que les systèmes de production et d'activité des agriculteurs familiaux en d'Afrique sub-saharienne sont diversifiés, et que cela permet une adaptation et une relative résilience face à ce type de choc.

\subsection{Des reconfigurations possibles dans la production et les circuits de distribution}

Les difficultés d'approvisionnement des marchés locaux rencontrées pour certains produits importés pourraient constituer une opportunité de développer la production locale. C'est par exemple la perspective qu'ouvre la fermeture des frontières du Maroc pour les producteurs d'oignons et d'orange d'Afrique de l'Ouest (Dugué, 2020). De même, la baisse des cours internationaux des produits de rente (cacao, coton, etc.) pourrait inciter les producteurs à reporter leurs efforts sur les cultures vivrières pour approvisionner les marchés locaux (Ruf, 2020; Dugué, 2020). Ce sont autant de stratégies d'adaptation qui pourraient transformer la crise liée à la Covid en opportunité de nouveaux revenus pour les producteurs et à une amélioration de la «souveraineté alimentaire», souhaitée par de nombreux gouvernements africains depuis la crise des prix internationaux de 2008, mais difficile à mettre en œuvre.

\section{De fortes contraintes sur l'accès à l'alimentation, en ville comme à la campagne}

L'arrêt ou la réduction des activités de nombreux secteurs économiques faisant vivre des millions de personnes en ville (restaurants, petit commerce, bâtiment, etc.) a aggravé la pauvreté et l'insécurité alimentaire. Dans les pays du Sud, la précarité alimentaire de nombre de travailleurs autoentrepreneurs, journaliers, du secteur «informel», d'ordinaire peu visibles dans des politiques de sécurité alimentaire centrées sur les ménages ruraux et le suivi de la campagne agricole, s'est fortement accentuée : selon les projections de l'IFPRI, c'est en Afrique que la pauvreté urbaine va surtout s'accentuer, plus qu'en Asie par exemple (Laborde et al., 2020). Dans ce numéro, Elloumi (2020) témoigne de l'augmentation des difficultés d'accès des populations les plus vulnérables à s'approvisionner en biens essentiels en raison de la crise Covid en Tunisie.

Les conséquences spécifiques de la Covid-19 et des mesures prises pour la contrer sur la sécurité alimentaire des familles de producteurs agricoles de l'Afrique semblent limitées à court terme (dans les 4 mois que l'on a considérés). Les producteurs ont (souvent, mais pas toujours) des réserves de céréales dans leurs greniers et/ou de tubercules dans les champs, et sont habitués à un certain niveau d'autonomie (très partielle, inégale, mais encore activable) (Dugué et al., 2021). Il est possible qu'ils ne soient pas tellement affectés pour le moment, étant habitués à des situations de crises «structurelles ». Ils ont souffert, ou vont peut-être moins souffrir, que les consommateurs urbains à court terme. En revanche, une incertitude forte demeure sur les moyen et long termes. En effet, l'agriculture familiale très importante en Afrique tire ses revenus à la fois de l'agriculture et d'autres activités exercées en milieu rural ou en ville, suivant des modèles migratoires variables vers d'autres zones rurales, des villes, d'autres pays africains, voire des pays développés (Mercandalli et Losch, 2018). Ces producteurs vont très certainement voir leur revenu non agricole reculer avec la récession économique qui se propage, et qui touche les opportunités non agricoles d'emploi local, comme les envois d'argent des migrants. De plus, la solidarité au sein des familles élargies pourrait s'inverser dans le sens des campagnes vers les villes. Ce sont les urbains qui vont demander de l'aide aux agriculteurs, soit par des envois de nourriture, soit en venant s'installer à la campagne. Il peut y avoir des tensions et ou des réorganisations dans les familles agricoles au sens large.

Plus généralement, cette crise met en avant l'importance qu'il convient d'accorder aux micro-, petites et moyennes entreprises, y compris dans l'agriculture et l'alimentation dans et après cette période de crise (von Braun et al., 2020). Elles pourvoient la plupart des emplois et des moyens d'existence des plus vulnérables en ville, et y contribuent grandement en milieu rural où la pluriactivité est la norme (Allen et al., 2018). 
De plus, dans le secteur agroalimentaire, ce sont elles qui assurent la transformation, le transport et la commercialisation des denrées et font fonctionner les filières alimentaires. Dans un contexte où les standards officiels sont peu nombreux ou peu respectés, la proximité relationnelle faite de multiples interactions interpersonnelles est la façon la plus répandue de faire respecter les contrats et de garantir la qualité entre ces acteurs des filières. Les mesures de restriction imposées dans différents pays (fermetures de marchés, limitation des déplacements, etc.) ont pour effet secondaire de possiblement affaiblir ces relations de proximité et de faire peser un risque sur le respect des contrats et de la qualité, au détriment des acteurs et actrices de ces filières parfois qualifiées d' «informelles» et peut-être au bénéfice des filières plus intégrées et standardisées (Moustier, 2013; Moustier et Nguyen, 2015). Il existe un réel risque de marginalisation et d'augmentation des inégalités à l'intérieur des filières ou tout au moins de reconfiguration entre les différents acteurs des filières d'approvisionnement alimentaires. Certains acteurs plus industriels, plus intégrés, souvent mieux organisés, risquent de gagner sur plusieurs tableaux : à la fois en gagnant des parts de marchés vis-à-vis de consommateurs rassurés par l'aspect standardisé des produits industriels, car les marchés ouverts et informels auront été plus souvent fermés que les supermarchés ou épiceries, et aussi car, souvent mieux organisés, ils auront eu l'écoute des gouvernements pour rester ouverts et approvisionner les populations. Les acteurs, plus nombreux et coordonnés dans des systèmes plus informels, risquent de perdre plus avec cette crise, car moins organisés et moins représentés dans les arènes politiques.

\section{Les politiques mises en place pour répondre à la crise}

Tous les pays ont pris des mesures de restriction de type confinement ou mise en quarantaine des zones affectées par le virus SARS-CoV2 pour endiguer sa propagation sur leurs territoires. Ces pratiques sont devenues une «norme» politique qui s'est étendue partout dans le monde au fur et à mesure que les pays étaient touchés, que ce soit en Asie, en Europe, en Afrique ou en Amérique latine. En Afrique comme ailleurs, des mesures de restriction ont été mises en place, et, en Afrique de l'Ouest en particulier, il faut rappeler que des mesures similaires avaient déjà été prises face à l'épidémie de la maladie à virus Ebola en 2014. À y regarder de plus près cependant, des différences notables apparaissent dans le degré de sévérité des mesures de restriction : confinement, distanciation sociale, télétravail, fermeture d'écoles, etc. (OCDE, 2020). Au Burkina Faso, par exemple, le gouvernement a décidé de fermer les marchés de plein air de la capitale, décision fortement critiquée alors que le voisin béninois était montré en exemple pour son souci de la mesure et de ne pas trop contraindre les activités économiques. Dans certains pays comme le Bénin en effet, le confinement n'a pas été choisi, car l'effondrement économique qui aurait pu résulter d'un confinement strict aurait pu avoir des conséquences plus graves que l'épidémie elle-même. C'est cette tension entre sauver des vies et protéger des moyens d'existence (livelihood) qui est complexe en situation d'incertitude et fait aujourd'hui l'objet de nombreux débats.
C'est pourquoi il est important de penser les adaptations possibles aux contextes locaux pour limiter les effets négatifs des mesures de restriction. Par exemple, la création de corridors sanitaires pour l'écoulement des denrées alimentaires ou le maintien de marchés de plein air avec des mesures sanitaires sont des mesures bien moins contraignantes que la fermeture des frontières et des marchés. Lors de la crise liée au virus Ebola en 2014 en Afrique de l'Ouest, les mêmes mesures de fermeture des frontières entre les pays touchés et de mise en quarantaine de villages ou de villes avaient provoqué d'importantes pertes post-récolte pour les filières de produits périssables. L'idée de corridors commerciaux associés à des contrôles sanitaires était déjà ressortie comme une recommandation forte de la part des acteurs des filières en 2014 (Alpha et Figuié, 2016). La constitution de stocks de sécurité pour les produits de base apparaît aussi particulièrement importante pour prévenir les ruptures d'approvisionnement (Carimentrand et Bah, 2020). Il reste difficile aujourd'hui de savoir quelles mesures ont été prises et lesquelles ont été efficaces.

Aujourd'hui, les pays mettent en place, également de façon généralisée, des mesures dites de soutien ou d'atténuation pour contrer les effets négatifs des premières mesures: soutien direct à certains secteurs d'activités, exonérations temporaires de taxes, filets sociaux de sécurité, etc. L'objectif est bien de relancer l'économie et de faire en sorte que les populations puissent de nouveau bénéficier de revenus ou d'aides spéciales (filets de sécurité) pour accéder aux biens et services indispensables, y compris l'alimentation.

Les capacités à financer et mettre en œuvre de telles mesures dépendent fortement du niveau de développement des pays; elles s'avèrent très vite limitées dans les pays pauvres, voire émergents, comme cela a pu être observé sur d'autres continents que l'Afrique. Là où une importante population urbaine est très précaire, comme au Brésil (Guéneau et Grisa, 2020) ou en Inde, les mesures de confinement ont entraîné des drames qui ont été compensés avec plus ou moins de succès et de façon différenciée au rythme des actions des villes, des États des structures fédérales et dans une certaine confusion. Cela n'est pas spécifique à ces pays, mais l'importance numérique des populations très précaires rend plus dommageable les tâtonnements des actions de protection sociale. À New Delhi en Inde par exemple, le gouvernement local a ouvert des écoles et a proposé des repas gratuits mais les migrants (les Indiens qui vivent une partie de l'année à la campagne et une autre partie en ville) étaient mal informés (Dorin, 2020). Dans des pays comme la Colombie, les mesures ciblant les producteurs ont semblé renforcer les inégalités, les agro-industriels et les producteurs d'exploitations moyennes ayant été beaucoup plus prompts à mobiliser les aides de l'État que les petits producteurs qui manquent d'accompagnement (Andrieu, 2020).

\section{Conclusion et perspectives pour la recherche}

Le tableau que l'on vient de présenter montre un grand contraste entre le monde de la production agricole et la sécurité alimentaire et nutritionnelle d'une grande partie des populations fragilisées. Le système alimentaire mondial a été peu 
perturbé. En revanche, les systèmes alimentaires locaux ont été fortement affectés par les mesures sanitaires de restriction en aval de la production, pour l'écoulement des produits sur les marchés internes ou d'export et, en bout de chaîne, au niveau des consommateurs qui ont vu leurs revenus diminuer et leur insécurité alimentaire et nutritionnelle s'aggraver.

Cette crise de la Covid met ainsi en exergue la déconnexion connue, mais particulièrement choquante dans ce contexte, entre une production agricole suffisante, un commerce mondial fonctionnel, un secteur agricole relativement protégé par rapport à d'autres secteurs économiques et l'accès limité, voire impossible, à l'alimentation pour des millions de personnes.

Les systèmes de protection sociale, d'assurance et d'aide ne sont pas suffisants pour assurer une continuité d'accès à l'alimentation pour tous, même si, dans de nombreux cas, les filières, marchés et magasins alimentaires ont été protégés par les gouvernements et les aliments ont été disponibles.

Les acteurs des filières alimentaires ont été ou sont affectés de façon différenciée à la fois par la récession économique liée aux mesures de restriction, et par les mesures de soutien prises par les gouvernements pour maintenir ou relancer l'activité économique. Ces dernières favorisent souvent les acteurs formels des filières au détriment des acteurs informels qui recouvrent la majorité des emplois de l'agroalimentaire dans les pays à faibles revenus. Sans appui aux petites et très petites entreprises, souvent informelles et maltraitées par les politiques publiques, un cercle vicieux s'enclenche où les petits commerçants et les transporteurs n'ont plus de travail, ne peuvent plus consommer, ce qui accélère la récession économique. Mais il existe des exemples où les gouvernements et/ou les acteurs ont réussi à préserver le système alimentaire « informel», comme en Côte d'Ivoire (Carimentrand et al., 2020).

Par ailleurs, les acteurs des systèmes alimentaires sont en train de développer des stratégies d'adaptation à la crise (report des cultures de rente vers le vivrier, nouveaux modes de consommation, etc.) dont on n'appréhende ni l'ampleur, ni les conséquences (Béné, 2020). Sont-elles conjoncturelles ou structurelles? Vont-elles renforcer la résilience et la durabilité de leur système? Sont-elles viables à long terme? Si un premier diagnostic pointe du doigt les défaillances qui poussent les plus vulnérables dans la précarité alimentaire, rien n'est certain quant aux conséquences à moyen et long termes de cette pandémie. Ces conséquences dépendront beaucoup de la capacité des acteurs à s'adapter ou se transformer, et de celle des politiques publiques à accompagner le changement.

Or, les politiques publiques se multiplient de façon souvent désordonnée, cherchant à répondre aux symptômes de la crise dans l'urgence sans chercher à intégrer les différentes composantes des systèmes alimentaires ou à accompagner les stratégies d'adaptation des acteurs. Il est crucial aujourd'hui d'adopter une approche systémique et multicritère pour penser ensemble production, transformation, distribution et consommation. Une telle approche doit également intégrer les différences spatiales et les questions spécifiques qu'elles posent, telles que la précarité alimentaire urbaine ou rurale, et les liens entre rural et urbain qui font vivre ces systèmes, les alimentent, les façonnent.

La gestion de la crise sanitaire doit ainsi viser non seulement à perturber le moins possible la fluidité des systèmes alimentaires, en privilégiant des mesures de restriction ciblées, mais aussi à les soutenir par des mesures inclusives destinées à l'ensemble des acteurs (achats publics aux producteurs, aides aux entreprises du secteur informel, protection des consommateurs les plus vulnérables, etc.). À plus long terme, la crise Covid fait ressortir de nombreuses pistes pour faire évoluer les systèmes alimentaires vers plus de résilience et de durabilité, que l'on peut regrouper en trois grands volets : (i) développer les systèmes alimentaires basés sur une diversification des systèmes de production par des pratiques agro-écologiques; outre les multiples avantages agronomiques, nutritionnels, sanitaires et environnementaux de ces systèmes, l'expérience montre que la diversité de la production est un atout clé pour permettre aux acteurs de rebondir après un choc (Audouin et al., 2020); (ii) développer les infrastructures et les technologies de stockage et de transformation pour les filières de produits périssables, afin de limiter les pertes post-récolte en cas de difficultés de commercialisation et ainsi de maintenir les revenus des acteurs des filières ; (iii) accompagner les micro- et petites entreprises des systèmes alimentaires, qui opèrent le plus souvent dans le secteur informel, pour répondre aux exigences croissantes de qualité (sanitaire et nutritionnelle) des produits finis et améliorer la qualité de leurs emplois. Ce soutien au secteur informel des systèmes alimentaires renvoie aussi à l'exigence d'un développement économique inclusif et ambitieux en matière de lutte contre les inégalités sociales et spatiales.

\section{Références}

AFDI. 2020. Les OP face à la crise du coronavirus. En Guinée, les producteurs de pomme de terre de la FPFD ne peuvent pas vendre leur récolte. AFDI. https://www.afdi-opa.org/les-op-face-a-lacrise-du-coronavirus-en-guinee-les-producteurs-de-pomme-deterre-de-la-fpfd-ne-peuvent-pas-vendre-leur-recolte/.

Allen T, Heinrigs P, Heo I. 2018. Agriculture, food and jobs in West Africa. West African Papers 14. Paris: OECD Publishing. https:// research4agrinnovation.org/wp-content/uploads/2018/06/ dc152bc0-en.pdf.

Alpha A, Figuié M. 2016. Impact of the Ebola virus disease outbreak on market chains and trade of agricultural products in West Africa. Dakar: FAO REOWA (Resilience, Emergencies and Rehabilitation in West Africa). http://www.fao.org/3/a-i5641e.pdf.

APESS (Association pour la promotion de l'élevage au Sahel et en Savane). 2020. Note d'analyse des premiers impacts de la pandémie du Covid 19 sur les exploitations familiales agropastorales membres de l'APESS. APESS, 28 p. https://www.apess.org/ wp-content/uploads/2020/04/Note-d\%E2\%80\%99analyse-des-pre miers-impacts-de-la-pand\%C3\%A9mie-du-COVID-19-sur-lesExploitations-Familiales-Agropastorales-membres-de-1\%E2\% 80\%99APESS.pdf.

Andrieu N. 2020. Covid-19 et sécurité alimentaire. En Colombie, la crise sanitaire exacerbe les inégalités entre petits et grands producteurs. [19/05/20]. https://www.cirad.fr/actualites/toutes-lesactualites/articles/2020/science/covid-19-securite-alimentairecolombie.

Audouin S, Bélières JF, Burnod P, David-Benz H, Vigne M, Rakotomalala W, et al. 2020. Covid-19 et sécurité alimentaire : à Madagascar, le lait ne s'écoule plus. [28/05/20]. https://www.cirad. fr/actualites/toutes-les-actualites/articles/2020/science/covid-secu rite-alimentaire-a-madagascar-la-filiere-lait-est-a-l-arret. 
Banque africaine de développement. 2020. Perspectives économiques en Afrique 2020 dans le contexte de la Covid-19. Supplément. Banque africaine de développement, 122 p. https://www.afdb.org/ fr/documents/perspectives-economiques-en-afrique-2020-supple ment? fbclid=Iw AR2 gsX1 c cyCKu 1-DBj439gwLx0 Cga cYdlXBxwz1 f068UYDTBfvlsqwQxmo.

Béné C. 2020. Resilience of local food systems and links to food security - A review of some important concepts in the context of COVID-19 and other shocks. Food Security 12: 805-822. DOI: 10.1007/s12571-020-01076-1.

Bricas N, Abadie C, Farrugia-Amoyel I, Lorieux A, Wood A. 2020. Solidarités alimentaires avec les populations précarisées par la crise du COVID-19 (phase 1). 5 p. http://www.chaireunesco-adm.com/ Solidarites-alimentaires-avec-les-populations-precarisees-par-lacrise-du-COVID.

Carimentrand A, Bah R. 2020. Covid-19 et sécurité alimentaire. Comment la Côte d'Ivoire protège ses systèmes alimentaires locaux. [21/04/20]. https://www.cirad.fr/actualites/toutes-les-actua lites/articles/2020/science/covid-19-securite-alimentaire-la-coted-ivoire-protege-ses-systemes-alimentaires-locaux.

CSA (Comité pour la sécurité alimentaire mondiale). 2012. S'entendre sur la terminologie. In: Trente-neuvième session du CSA, 15-20 octobre 2012. Rome: CFS 2012/39/4.

Daviron B. 2012. Prix internationaux des produits alimentaires: volatilité ou hausse durable? Implications pour le débat international. Revue Tiers Monde 211(3): 91-109. DOI: 10.3917/rtm.211.0091.

Dorin B. 2020. Covid-19 et sécurité alimentaire: 1'Inde face à la crise de l'emploi. [15/04/20]. https:/www.cirad.fr/actualites/toutes-lesactualites/articles/2020/science/covid-19-securite-alimentaire-1inde-face-a-la-crise-de-1-emploi.

Dugué P. 2020. Covid-19 et sécurité alimentaire. Pourquoi l'agriculture africaine se montre résiliente face à la crise. https:// www.cirad.fr/actualites/toutes-les-actualites/articles/2020/science/ covid-19-securite-alimentaire-1-agriculture-africaine-resilienteface-a-la-crise.

Dugué $\mathrm{P}$, Kohio E, Tiemtoré J. 2021. L'agriculture burkinabè face à la crise de la Covid-19: cas des régions du Yatenga et des HautsBassins. Cahiers Agricultures 30. DOI: 10.1051/cagri/2021002.

Elloumi M. 2020 L'agriculture tunisienne face à la Covid-19 : impacts de la crise sanitaire et perspectives pour une agriculture résiliente. Cahiers Agricultures 29: 35. DOI: 10.1051/cagri/2020032.

FAO. 2020. Sustainable food production and COVID-19. FAO. http:// www.fao.org/3/ca8807en/CA8807EN.pdf.

FAO, IFAD, UNICEF, WFP, WHO. 2020. Transforming food systems for affordable healthy diets. In: Brief to the state of food security and nutrition in the world 2020. Rome: FAO. DOI: 10.4060/ ca9699en.

FPFD (Fédération des paysans du Fouta Djallon). 2020. Note d'impact du virus Covid-19 sur les actions de la FPFD et ses membres. FPFD.

FMI (Fonds monétaire international). 2020. World economic outlook update. FMI, 20 p. [06/20]. https://www.imf.org/en/Publications/ WEO/Issues/2020/06/24/WEOUpdateJune2020.

Independent Expert Group. 2020. Global nutrition report. Action on equity to end malnutrition. Bristol, UK: Development Initiatives. https://globalnutritionreport.org/reports/2020-global-nutritionreport/.

Guéneau S, Grisa C. 2020. Covid-19 et sécurité alimentaire: au Brésil, l'aide d'urgence suffira-t-elle à garantir l'accès à l'alimentation pour les plus démunis? [24/04/20]. https://www. cirad.fr/actualites/toutes-les-actualites/articles/2020/science/ covid-19-securite-alimentaire-bresil.
HLPE. 2017. Nutrition et systèmes alimentaires. Rapport du Groupe d'experts de haut niveau sur la sécurité alimentaire et la nutrition du Comité de la sécurité alimentaire mondiale. Rome: FAO. http:// www.fao.org/3/I7846FR/i7846fr.pdf.

IPES Food. 2020. Le Covid-19 et la crise dans les systèmes alimentaires : symptômes, causes et solutions potentielles. IPES Food. [04/20]. http://www.ipes-food.org/_img/upload/files/ COVID19\%20COMMUNICATION_FR.pdf.

Laborde Debucquet D, Mamun A, Parent M. 2020. Documentation for the COVID-19 food trade policy tracker: tracking government responses affecting global food markets during the COVID-19 crisis. COVID-19 Food Trade Policy Tracker Working Paper 1. Washington, DC: International Food Policy Research Institute (IFPRI). DOI: $10.2499 / \mathrm{p} 15738$ coll2.133711.

Laborde D, Vos R, Martin W. 2020. Poverty and food insecurity could grow dramatically as COVID-19 spreads. IFPRI Blog, research post. DOI: $10.2499 /$ p15738coll2.133762_02.

Lao Farmer Network. Impact of COVID-19 on farmers in Laos. Lao Farmer Network. [03/04/20]. https://www.youtube.com/watch?v= gTbGbQjEphk.

MAA (Ministère de l'Agriculture et de l'Alimentation), République française. 2020. Veille agroalimentaire. Afrique de l'Ouest et Afrique centrale. $\mathrm{n}^{\circ} 5$. Note manuscrite. Ministère de l'Agriculture et de l'Alimentation.

Mendez Del Villar P. 2020. Le ralentissement de l'économie lié au Covid-19 risque de provoquer une crise généralisée de la demande. [05/20]. https://www.cirad.fr/actualites/toutes-les-actualites/arti cles/2020/questions-a/patricio-mendez-del-vilar-covid-19-etrisque-de-crise-generalisee-de-la-demande.

Mercandalli S, Losch B, eds. 2018. Une Afrique rurale en mouvement. Dynamiques et facteurs des migrations au sud du Sahara. Rome: FAO et CIRAD, 60 p. https://www.cirad.fr/MM/ atlas-fao/ATLAS_A_moving_rural_Africa_FR.pdf.

Moustier P. 2013. Reengaging with customers: proximity is essential but not enough. Acta Hortic 1006: 17-33. DOI: 10.17660/ ActaHortic.2013.1006.1.

Moustier P, Nguyen TTL. 2015. The role of proximity and standards in guaranteeing vegetable safety in Vietnam. World Food Policy 2 (1): 52-77. DOI: 10.18278/wfp.2.1.4.

DeParle P. 2020. As hunger swells, food stamps become a partisan flash point. The New York Times. [07/05/20]. https://nyti.ms/ 2A6fyhw.

OCDE. 2020. Key policy responses from the OECD. OCDE. https:// www.oecd.org/coronavirus/en/policy-responses.

Organisation internationale du travail. 2020. ILO monitor: COVID19 and the world of work, third edition updated estimates and analysis. Organisation internationale du travail. [29/04/20]. https:// www.ilo.org/wcmsp5/groups/public/-dgreports/-dcomm/docu ments/briefingnote/wcms_743146.pdf.

RPCA (Réseau de prévention des crises alimentaires). 2020. Impact de la crise du Covid-19 sur la sécurité alimentaire et nutritionnelle au Sahel et en Afrique de l'Ouest. Note d'information et de veille $\mathrm{n}^{\mathrm{o}}$ 1. RPCA. [04/2020]. http://www.food-security.net/wp-content/ uploads/2020/05/Note-aux-decideurs_SAN_covid-N1.pdf.

Ruf F. 2020. Covid-19 et sécurité alimentaire. Les planteurs de cacao de Côte d'Ivoire craignent une baisse de leurs revenus. [07/05/20]. https://www.cirad.fr/actualites/toutes-les-actualites/articles/2020/ science/covid-19-securite-alimentaire-quels-risques-pour-les-plan teurs-de-cacao-ivoiriens.

Vall E, Corniaux C, Diaw A, Seck D, Sall C, Vigne M, et al. 2020a. Effet de Covid-19 sur la production et la commercialisation du lait en Afrique : les cas de Madagascar, du Burkina Faso et du Sénégal. 
Africa-Milk. [27/05/20]. https:/www.africa-milk.org/fr/actualites/ effet-de-covid-19-sur-la-production-et-la-commercialisation-du-laiten-afrique-les-cas-de-madagascar-du-burkina-faso-et-du-senegal.

Vall E, Mburu J, Ndambi A, Sall C, Diaw Camara A, Sow A, et al. $2020 \mathrm{~b}$. First effects of the COVID-19 outbreak on the African dairy industry: cases of Kenya, Madagascar, Burkina Faso and Senegal. Cahiers Agricultures 29. DOI: 10.1051/cagri/2020047.

von Braun J, Zamagni S, Sorondo MS. 2020. The moment to see the poor. Science 368(6488): 214-214. DOI: 10.1126/science. abc2255.

Citation de l'article : Dury S, Alpha A, Zakhia-Rozis N, Giordano T. 2021. Les systèmes alimentaires aux défis de la crise de la Covid-19 en Afrique: enseignements et incertitudes. Cah. Agric. 30: 12. 